\title{
émulations
}

\section{La nuit urbaine, un espace-temps complexe entre opportunités et inégalités}

\author{
Hélène Jeanmougin et Emanuele Giordano
}

Émulations - Revue de sciences sociales

2020, $n^{\circ} 33$, "La nuit urbaine. Un espace-temps complexe entre opportunités et inégalités ».

\section{Article disponible à l'adresse suivante}

https://ojs.uclouvain.be/index.php/emulations/article/view/jeanmougin

\section{Pour citer cet article}

Hélène Jeanmougin et Emanuele Giordano, « La nuit urbaine, un espace-temps complexe entre opportunités et inégalités », Émulations, n³3, Mise en ligne le 15 juin 2020.

DOI : 10.14428/emulations.033.01

Distribution électronique : Université catholique de Louvain (Belgique) : ojs.uclouvain.be

(C) Cet article est mis à disposition selon les termes de la Licence Creative Commons Attribution, Pas d'Utilisation Commerciale 4.0 International. http://creativecommons.org/licenses/by-nc/4.0/

Éditeur : Émulations - Revue de sciences sociales / Presses universitaires de Louvain https://ojs.uclouvain.be/index.php/emulations

ISSN électronique : 1784-5734

PUL PRESSES

UNIVERSITAIRES

DE LOUVAIN 


\title{
La nuit urbaine, un espace-temps complexe entre opportunités et inégalités
}

\author{
Hélène Jeanmougin ${ }^{1}$ et Emanuele Giordano ${ }^{2}$
}

\begin{abstract}
[Résumé] L'appropriation de la nuit urbaine par des activités économiques, sociales et culturelles a connu au XXe siècle une accélération sans précédent. L'intérêt croissant des sciences sociales pour cet objet de recherche a permis de mieux connaître les dynamiques caractérisant l'évolution de l'espace-temps nocturne dans les différentes parties du globe. Cette introduction souligne la nécessité de poursuivre l'exploration de cet espace-temps complexe. Ce numéro, en réunissant des contributions de différentes disciplines et sur des régions géographiques variées, propose de lier tendances globales et spécificités locales qui (re)configurent la nuit urbaine dans la ville contemporaine. Les apports de ces contributions sont présentés et articulés autour de quatre axes: lieux et usages différenciés des pratiques nocturnes, attractivité et conflits, inégalités nocturnes, méthodologies des enquêtes sur la nuit.
\end{abstract}

Mots-clés : nuit urbaine, pratiques nocturnes, inégalités nocturnes, conflits liés à la nuit.

The urban night, a complex space-time between opportunities and inequalities

[Abstract] The appropriation of the urban night by economic, social and cultural activities has experienced an unprecedented acceleration in the 20th century. The growing interest of the social sciences in this research object has produced a vast knowledge on the dynamics that characterize the evolution of the nighttime in different parts of the globe. This introduction highlights the need to continue exploring this complex spacetime. This issue brings together contributions from different disciplines and focus on various geographic regions, thus making it possible to explore global trends and local specificities which (re)configure the urban night in the contemporary city. These contributions are presented and articulated in four axes: differentiated places and uses of nocturnal practices, attractiveness and conflicts, nocturnal inequalities, methodology of night surveys.

Keywords: urban night, night practices, inequalities, urban conflicts.

\section{Introduction}

Dans les dernières décennies, l'espace-temps nocturne a connu de profondes modifications. Si l'appropriation progressive de la nuit urbaine par un nombre croissant d'activités économiques, sociales et culturelles est un phénomène historique de longue durée (Koslofsky, 2011), ce processus a connu au XX siècle une accélération sans précédent. L'une des principales conséquences de la transformation progressive des grands rythmes urbains traditionnels par le processus de néolibéralisation a en effet conduit à la nocturnalisation d'un nombre croissant de pratiques de production et de consommation (ouverture prolongée de certains commerces, fonctionnement nocturne de transports publics, etc.). Cependant, à l'exception de travaux pionniers (Cauquelin,

\footnotetext{
${ }^{1}$ Lames, CNRS, Aix-Marseille Université, France.

${ }^{2}$ Laboratoire Babel EA 2649, Université de Toulon, France.
} 
1977 ; Melbin, 1978), l'intérêt des sciences sociales à l'égard de la nuit urbaine ne s'est développé que récemment, la nuit urbaine n'ayant jusqu'alors reçu qu'un intérêt marginal des chercheurs. Ce n'est qu'à partir de la seconde moitié des années 1990 que la nuit urbaine a commencé à être intégrée aux débats scientifiques sur la ville contemporaine.

Ce débat a d'abord porté sur le concept d'économie nocturne, la Night-Time-Economy (NTE), c'est-à-dire les activités économiques se déroulant pendant la soirée et la nuit. Utilisé aujourd'hui mondialement, ce concept trouve son origine dans un moment historique et dans un contexte géographique précis : les villes du nord de l'Angleterre au début des années 1990. Dans le cadre des politiques de déréglementation impulsées par les gouvernements conservateurs, la diffusion de ce concept fait référence aux travaux des membres des think tanks tels que Comedia, visant à encourager la déréglementation des règles sur les débits de boissons (Bianchini, 1995), alors très contraignantes. Le but était de développer des espaces urbains dédiés aux pratiques des jeunes dans les bars, les pubs, les boîtes de nuit et les salles de concert.

La stratégie de la ville active « 24 heures sur 24 » était considérée pour les aménageurs comme une possible réponse à la suburbanisation progressive de la vie urbaine (Heath, 1997). L'économie de la nuit constituait une opportunité pour revitaliser des centresvilles qui, à l'époque, souffraient d'un manque de sécurité ; il s'agissait ainsi d'attirer de nouvelles populations dans le centre-ville pendant les soirées et la nuit (Lovatt, O’Connor, 1995). La NTE était donc l'occasion de « doubler » l'économie de la ville, en doublant la période de temps dans laquelle les centres-villes étaient productifs.

Alors qu'initialement le développement du concept de NTE était relié aux conditions sociopolitiques spécifiques existantes au Royaume-Uni, il est maintenant devenu, à différents degrés de développement en fonction des divers contextes nationaux, un phénomène mondial, comme le souligne Ilse Van Liempt et al. :

Originaires des villes postindustrielles du Royaume-Uni, les stratégies visant à (re)positionner la nuit urbaine en termes d'opportunités économiques et de revitaliser les centres urbains, qui sont soutenus par les rationalités de la compétitivité urbaine dans une économie mondialisée, ont régulièrement été diffusées à travers l'Europe, en Amérique du Nord et en Australasie et sont maintenant poursuivies, bien que sous une forme modifiée, dans les villes du Sud global (Van Liempt, 2015 : 409-410).

Si la NTE était préalablement associée à la volonté de revitaliser des centres urbains délaissés, à partir des années 2000, plusieurs chercheurs ont commencé à souligner la façon dont la NTE, en l'absence de véritables politiques de contrôle et de gestion, pouvait avoir des résultats désastreux pour la santé des consommateurs et favoriser l'émergence de conflits entre différentes populations urbaines (Hadfield, 2009). L'optimisme initial au sujet du potentiel offert par la NTE s'est peu à peu dissipé. Aujourd'hui, la littérature s'accorde sur le fait que la NTE, dans toutes ses formes et spatialités, se caractérise par de profondes discriminations en termes de classe, de sexe, d'âge et de race (Nofre, Eldridge, 2018). 
Si les études sur la NTE constituent toujours la part la plus consistante des études sur la nuit urbaine, des travaux plus récents critiquent cependant ces premières perspectives de recherche qui réduiraient la nuit urbaine aux bars, pubs et restaurants, ainsi qu'aux rationalités économiques qui sous-tendent leurs développements (Shaw, 2015a). Une série de nouvelles études propose une approche plus holistique de la nuit urbaine, en explorant la multiplicité des pratiques culturelles, sociales et économiques qui caractérisent la ville nocturne (Gwiazdzinski, 2005 ; Straw, 2015). La nuit urbaine est ainsi devenue un thème émergent dans les sciences sociales francophones, comme le montre une série de travaux récents en sociologie (Guérin, 2016 ; Jeanmougin, 2019), en géographie (Pieroni, 2017 ; Giordano, 2017 ; Comelli, 2015), ou en aménagement (Bertin, 2016), mais aussi dans les sciences sociales anglophones (Roberts, Eldridge, 2009), espagnoles (Nofre, 2008) et italiennes (Giordano, Gwiazdzinski, 2018). Plusieurs numéros de revue récemment consacrés à cet objet (Candela, 2017 ; Gwiazdzinski, Pignot, Saez, 2019 ; Gwiazdzinski, Maggioli, Straw, 2019 ; Monod Becquelin, Galinier, 2020) soulignent également son importance croissante dans les sciences sociales.

De nouveaux objets de recherche viennent enrichir ce champ, comme l'urbanisme temporel (Mallet, 2014), la géographie de la pollution lumineuse (Challéat, 2010) ou les recherches qui établissent un lien entre vie nocturne et gentrification (Jeanmougin, Bouillon, 2016 ; Nofre et al., 2017). Certains travaux, aux visées plus opérationnelles, interrogent la diversité d'usages et de mobilités nocturnes pour proposer des transports plus adaptés à cette diversité (Espinasse, Buhagiar, 2004), tandis que d'autres explorent le rôle de l'éclairage urbain (Deleuil, Toussaint, 2000) ou élaborent des cartographies des nuisances sonores nocturnes en plaidant pour des villes aux décibels moins élevés (Radicchi, 2017). Enfin, les études sur la nuit sont désormais sorties des limites de la ville et ont commencé à s'intéresser aux expériences nocturnes des espaces périurbains (Didier-Fèvre, 2018) et ruraux (Kumar, 2015).

L'ensemble de ces travaux de recherche permet d'appréhender la croissante complexité de la temporalité nocturne et de dépasser une vision stéréotypée de la nuit comme un temps homogène, en produisant une connaissance plus nuancée et critique des dynamiques qui caractérisent l'évolution de l'espace-temps nocturne dans les différentes parties du globe. À l'échelle globale, on peut observer - avec des degrés de développement différents - la multiplication des activités nocturnes et l'extension des heures d'ouverture d'une série de services et activités traditionnellement diurnes tels que les musées et les bibliothèques, jusqu'à arriver au fonctionnement $24 \mathrm{~h}$ sur $24 \mathrm{~h}$ de certains services comme le transport public (Mallet, 2014). Si l'extension des activités diurnes ne concerne pas l'ensemble des espaces, elle progresse, de façon discontinue, avec des différences substantielles persistant entre espaces urbains et ruraux, centre et périphérie. Il ne s'agit pourtant pas de se saisir de cet objet de façon duale en appréhendant la nuit uniquement comme l'envers du jour, puisque «l'expérience de ceux qui utilisent la nuit publique de diverses manières, par choix ou par nécessité, révèle un espace-temps caractérisé par une vie sociale significative et indépendante de l'expansion 
du jour » (Shaw, 2015b : 645). La nuit reste ainsi, spatialement et fonctionnellement, un espace-temps à l'existence à la fois singulière et plurielle.

Le résultat de cette croissante complexité de pratiques, représentations et intérêts est l'émergence d'un espace-temps contrasté, voire inégalitaire. Si la nuit est porteuse de nouvelles opportunités économiques (Giordano et al., 2018), tout comme d'opportunités de proposition et d'expression culturelle, sociale ou politique comme le montre le mouvement Nuit debout en France (Shaw, 2017), elle reste aussi un espace-temps caractérisé par des inégalités profondes. Au-delà des travaux déjà cités sur la NTE, une série d'études a montré les formes d'exploitation qui caractérisent la croissance du travail nocturne et ses impacts négatifs sur la santé et la vie sociale des travailleurs (Macarie, 2017). D’autres travaux encore ont commencé à analyser de quelles façons l'ouverture des activités commerciales la nuit tend à favoriser la grande distribution et les activités commerciales dans le centre-ville par rapport à celles des banlieues (Mallet, 2009). Ainsi, selon certains auteurs, la nuit est aujourd'hui caractérisée par une « citoyenneté discontinue »(Gwiazdzisnki, 2007) en raison du fait qu'une partie importante de la population n'a pas accès, durant les heures nocturnes, à certains droits fondamentaux comme le droit à la mobilité. La progressive marchandisation des loisirs nocturnes tout comme les dynamiques de distinction au sein de certains lieux de fréquentation nocturne (Pinçon, Pinçon-Charlot, 2000) peuvent également être excluantes pour les populations les moins dotées en capital financier et/ou social. La dimension de genre joue aussi un rôle. Plusieurs travaux explorent par exemple les stratégies mises en place par les femmes lors des pratiques et déplacements nocturnes (Lieber, 2011 ; Bernard-Hohm, Raibaud, 2012). Par ailleurs, la nuit reste une temporalité particulièrement hostile pour plusieurs populations urbaines (travailleuses du sexe, SDF, etc.), rarement considérées dans la recherche urbaine ni dans l'élaboration des politiques sur la ville nocturne.

Malgré un intérêt croissant pour cet objet, la métaphore de la nuit comme « dernière frontière de la ville » ou « nouvel espace à conquérir » (Gwiazdzinki, 2005) reste valable dans le champ de la recherche académique : la nuit est un espace-temps complexe et fragmenté, dont les dynamiques et les tensions restent encore largement à examiner par les sciences sociales contemporaines. L’objectif de ce numéro thématique est de contribuer à l'exploration croissante de la frontière nocturne par les sciences sociales en permettant d'apprécier la complexité de cet espace-temps. Il réunit des contributions de chercheurs issus de différentes disciplines, travaillant sur différentes régions géographiques, et contribuant à l'émergence et à la richesse de cet objet de recherche. Les quatre axes de réflexion de ce numéro proposent d'articuler les tendances globales et les spécificités locales qui (re)configurent la nuit urbaine dans la ville contemporaine, en se saisissant de quelques-unes des entrées par lesquelles il est possible d'appréhender cet espace-temps spécifique. 


\section{Les pratiques nocturnes : des lieux et des usages différenciés}

Si les usages jeunes et festifs de la nuit urbaine sont assez bien documentés (Chatterton, Hollands, 2003 ; Guérin, 2016 ; Nofre et al., 2016), les pratiques nocturnes se résument-elles à un usage récréatif et consumériste réservé à une population jeune ? Si les bars, restaurants et discothèques sont des lieux classiques de sociabilités nocturnes, d'autres lieux peuvent être les supports de ces sociabilités, à l'instar de ces snacks égyptiens marseillais permettant des rencontres inédites entre consommateurs issus de mondes sociaux bien différenciés (Bouillon, 2000). La nuit est également propice à des activités illégales ou clandestines, mobilisant discrétion et labilité des lieux et des présences : nous pensons par exemple à la pratique du graffiti, au deal, aux squats ou aux rencontres sexuelles clandestines (Gaissad, 2000). Comment s'articulent alors ces pratiques et ces lieux à cet espace-temps spécifique?

L'article de Gwenaëlle Dourthe et Alexandre Grondeau se penche ainsi sur l'existence de free parties dans lesquelles la dimension festive s'oppose à la croissante mercantilisation des lieux nocturnes festifs s'inscrivant dans un " néolibéralisme territorial ». $\grave{A}$ travers l'exploration des pratiques socioculturelles et territoriales alternatives qui caractérisent ces rassemblements festifs illégaux, les auteurs mettent à jour la création de « zones temporaires autonomes (TAZ) » (Bey, 1991) où s'invente un « droit à la ville libre et festive ».

À Dakar, les « aventurières de la nuit», sur lesquelles portel'article de Thomas Fouquet, fréquentent quotidiennement des bars et night-clubs de la capitale sénégalaise à la recherche de destins plus enviables dont la réalisation passerait notamment par la migration. Se basant sur une longue et minutieuse ethnographie de leurs nuits dakaroises, l'auteur montre comment ces jeunes femmes perçoivent et investissent « le Dakar-by-night » comme une scène expressive où d'autres destins sont susceptibles de trouver un mode de concrétisation ou au moins de figuration, ne serait-ce qu'au travers de « mobilités ascendantes fictives ».

L'article d'Arianna Cecconi s'intéresse, quant à lui, à des femmes vivant le revers de l'expérience migratoire si convoitée par ces jeunes femmes dakaroises, et à l'altération du sommeil qui en découle. S’inscrivant dans les recherches appréhendant le sommeil et les rêves comme une perspective pour étudier la société (Bilhaut, 2003 ; Galinier et al., 2010 ; Lahire, 2018), l'article interroge les décalages entre les normes de sommeil occidentales et les rythmes et les habitudes de sommeil de plusieurs mères d'enfants en bas âge vivant dans un quartier marseillais socialement défavorisé. À travers l'exploration des difficultés d'endormissement de ces femmes, Arianna Cecconi analyse la façon dont les idées normatives sur le sommeil produisent des formes de vie qui sont considérées à la fois comme un signe de santé ou de désordre, créant chez ces femmes une pression à se soumettre à ce référent de la «normalité ». 


\section{Vie nocturne animée : entre attractivité et conflits}

Dans un contexte de compétition interurbaine au niveau international, l'offre de divertissement nocturne est devenue un atout pour les municipalités souhaitant attirer touristes et jeunes consommateurs (Roberts, Eldridge, 2009). Si une vie nocturne animée est ainsi un facteur d'attractivité urbaine, elle peut cependant s'accompagner d'effets indésirables (nuisances nocturnes, développement d'une monoactivité commerciale de divertissement nocturne au détriment des commerces de proximité, pénurie de logements résidentiels due à l'essor des locations touristiques, etc.). Si certaines villes comme Berlin ou Barcelone (Nofre et al., 2018) subissent les conséquences de ces effets indésirables depuis déjà plusieurs années, ils sont observables également dans des contextes urbains plus modestes comme Bordeaux (Comelli, 2015). Quels sont les objets et les enjeux de ces conflits ? Quels acteurs impliquent-ils ? Quels sont les effets locaux ambivalents d'une vie nocturne animée pour un territoire ? De quelles manières les municipalités ou les résidents s'en emparent-ils ? Quelles sont les politiques urbaines mises en œuvre pour, d'une part, souligner l'attractivité nocturne locale et, d'autre part, en limiter les effets indésirables?

C'est à ces différentes interrogations que répondent les textes d'Adrien Defrance et d'Emanuele Giordano et Pedro Gomes. Prenant pour contexte la rue Jean-Pierre Timbaud à Paris, l'article d'Adrien Defrance permet d'appréhender plusieurs dimensions d'un conflit autour du «bruit récréatif nocturne » existant dans cette rue. L'auteur apporte des éclairages à la fois sur les critères faisant varier les perceptions de ce bruit par les résidents, sur les enjeux des mobilisations des différents acteurs liés à ce conflit (habitants, sortants nocturnes, gérants de bar), et sur la difficulté, pour les acteurs institutionnels (responsables d'associations, élus, médiateurs), de trouver des formes d'aménagement de l'espace public et des modalités de médiation permettant de satisfaire différents groupes aux intérêts et motivations divergents.

Emanuele Giordano et Pedro Gomes interrogent, quant à eux, les relations qui existent entre l'aménagement urbain et le développement des loisirs nocturnes dans deux contextes urbains, celui du quartier de l'Écusson à Montpellier et celui du Cais do Sodré à Lisbonne. Tout en montrant que l'existence d'une vie nocturne animée est valorisée par ces deux municipalités, ils explorent et analysent les cohabitations difficiles entre résidents et noctambules. Ils interrogent les productions et les régulations urbanistiques des espaces publics en soulignant que leurs impensés et leurs ambiguïtés en termes d'usages et de temporalités jouent un rôle crucial dans les conflictualités observées entre « ville qui dort » et « ville qui sort ».

L'article de Gwenaëlle Dourthe et Alexandre Grondeau sur le mouvement free party souligne également la valeur heuristique du conflit. Il rappelle en effet que c'est souvent à partir de situations conflictuelles que naissent les contre-cultures et les pratiques festives alternatives, se construisant dans les interstices des règles et des principes politiques auxquels elles s'opposent. Refusant les discriminations socioéconomiques, raciales ou vestimentaires inhérentes à la mercantilisation des lieux nocturnes festifs, 
ce mouvement s'est étendu à toute l'Europe suite au départ d'une partie des premiers « teuffeurs » du Royaume-Uni à cause de l'intense répression gouvernementale concernant l'encadrement du monde de la nuit, l'inculpation des premiers « teuffeurs britanniques » pour « conspiracy to cause public nuisance » et l'établissement des lois du Criminal Justice and Public Act en 1994.

\section{La nuit, facteur d'inégalités}

La complexification progressive de la nuit urbaine provoquée par la désynchronisation des rythmes de vie produit un nombre croissant d'inégalités sociales et spatiales (Mallet, 2014), venant souvent se superposer à celles déjà existantes dans la journée. Le manque d'accessibilité aux infrastructures et services urbains nocturnes renforce par exemple les inégalités entre centre et périphérie, et entre classes sociales aisées et populaires. Quelles sont donc les différentes discriminations sociales et spatiales produites par cet espace-temps spécifique, et les manières de les contourner ou de les détourner?

L'article de Gwenaëlle Dourthe et Alexandre Grondeau répond partiellement à cette question, en appréhendant la free party, ses usagers et les territoires qu'ils génèrent comme un moyen de lutter contre les discriminations socioéconomiques, culturelles ou vestimentaires inhérentes à la marchandisation des lieux nocturnes festifs. Si les nuits de ces « teuffeurs » sont bel et bien festives, elles le sont hors d'un cadre consumériste établi, au sein de TAZ (zones autonomes temporaires) où ont cours d'autres normes et valeurs. Ce faisant, l'exemple du mouvement free party démontre les « compétences » des individus à infléchir (au moins partiellement) le cours des choses à travers « un refus de se couler strictement dans les propositions faites par la puissance publique » (Deboulet et al., $2007: 10$ ).

En opposant " la fluidité " associée aux positions occupées ou accessibles durant la nuit à « la rigidité » des systèmes de contraintes (économiques, sociales, morales) diurnes et à la dureté des conditions de vie à Dakar, Thomas Fouquet souligne, quant à lui, les opportunités dont est porteuse la « liquidité du nocturne » pour les jeunes femmes dakaroises enquêtées, à l'avenir incertain et au présent caractérisé par « un désir d'Ailleurs » spatial et social. Il montre comment celles-ci accumulent des compétences, des savoir-être et des savoir-faire spécifiques à l'espace-temps nocturne au fil de constructions de " carrières de la nuit ", qui permettraient, selon l'auteur, d'avoir davantage prise sur sa propre trajectoire et de la négocier au plus près de ses aspirations et intérêts.

Si la nuit est porteuse d'opportunités pour ces jeunes aventurières nocturnes dakaroises, l'espace-temps nocturne peut également se caractériser par de fortes contraintes de genre, comme le montre Marie Bonte dans son article. À travers des réflexions méthodologiques sur son terrain doctoral - les nuits beyrouthines -, la chercheuse met à jour les difficultés qu'elle a dû y affronter et qui existent plus largement pour les jeunes femmes de la capitale : nécessité de respecter les « codes de la bienséance et de normes 
de respectabilité par rapport aux hommes ", épisodes récurrents de drague en tant que consommatrice dans un bar ou d'inconfort en tant que serveuse face à certains clients hommes (" pour qui être au service signifie être à disposition»), ou encore peur face au danger d'être abusée sexuellement.

Plusieurs travaux explorent les stratégies mises en place par les femmes face à ces contraintes de genre lors des pratiques et déplacements nocturnes (Lieber, 2011 ; Bernard-Hohm, Raibaud, 2012), mais peu d'auteurs s'intéressent aux perceptions et aux expériences féminines du sommeil. C'est l'objet de l'article d'Arianna Cecconi, qui s'intéresse au sommeil urbain en tant que sujet social, influencé par des représentations, des variations matérielles et environnementales, ainsi que par les inégalités sociales. À partir d'une ethnographie réalisée auprès de mères d'enfants en bas âge vivant dans un quartier marseillais socialement défavorisé, l'auteure explore les décalages entre une conception normative du sommeil véhiculée notamment par le corps médical et la diversité culturelle de « techniques du sommeil » (Mauss, 1936) de ces femmes, dont la plupart ont connu une expérience migratoire. Elle questionne également la pertinence de ces normes de sommeil pour ces femmes et leurs enfants dans un environnement socio-urbain se caractérisant par de forts taux de chômage, des logements vétustes et surpeuplés, des espaces publics inhospitaliers et une charge parentale assurée quasi exclusivement par les femmes, soulignant in fine l'enjeu avant tout social et politique du sommeil.

\section{Méthodologies}

Si les perceptions des espaces et des temporalités nocturnes sont bien distinctes de celles diurnes, étudier la nuit implique-t-il également des méthodologies spécifiques? L'article de Marie Bonte nous invite à une réflexion sur les contraintes et les adaptations méthodologiques que nécessitent des terrains de recherche nocturnes. Ainsi, pour l'auteure, faire du terrain la nuit implique tout à la fois une inversion des rythmes biologiques, une modification des perceptions, et une accentuation du risque (réel, perçu ou construit), qui conditionnent à la fois l'accès à certains endroits et les choix méthodologiques. Un des intérêts de l'article est de souligner comment ces contraintes liées à la dimension nocturne peuvent devenir des ressources grâce, par exemple, à des rapprochements éphémères et plus spontanés que dans les entretiens semi-directifs ou à la compréhension des rapports sociaux de sexe, mais aussi de la place du désir et de la sexualité sur le terrain qui, « une fois remis à la bonne distance réflexive », ont significativement augmenté sa compréhension de la jeunesse noctambule de Beyrouth. Via la notion de " distance »- entendue notamment « comme un curseur que l'on ajuste en fonction des situations » et des postures adoptées - elle analyse la mise en place simultanée de plusieurs méthodes d'observation du monde de la nuit et de la délimitation du terrain, en soulignant que ces deux processus nécessitent de composer avec la place que les acteurs nous assignent. L'hétérogénéité et la fluctuation des rôles sociaux et des identités, que cette chercheuse a vécues et analyse ici, ne sont pas sans rappeler la 
relative confusion que l'espace-temps nocturne induit quant aux rôles et statuts individuels des « aventurières de la nuit dakaroises » de l'article de Thomas Fouquet autour de la « liquidité » nocturne.

Dans leur article, Emanuele Giordano et Pedro Gomes présentent une stimulante approche croisée et comparative des pratiques urbaines et de leurs régulations : l'une des enquêtes partant des pratiques urbaines pour ensuite questionner leur prise en compte par l'action publique, l'autre partant de l'action publique pour questionner la manière dont elle régule certaines pratiques urbaines. La diversité et la complémentarité méthodologiques (entretiens avec des décideurs des politiques urbaines, observations des pratiques urbaines, recensement et analyse des décrets réglementant la vie nocturne, analyse d'articles de presse) de cette approche croisée, tout en mettant en évidence les points communs observés dans plusieurs contextes urbains, permettent de montrer comment la nuit peut être un espace-temps privilégié pour analyser plus largement certaines des ambiguïtés et contradictions qui caractérisent les politiques de revitalisation des centres historiques des villes européennes.

Enfin, à travers la notion d'« imagination participante », Arianna Cecconi propose une méthodologie originale qui mêle approche anthropologique, entretiens semi-directifs, pratique artistique et collaborations avec des médecins et psychologues. En considérant cette recherche comme étant également une possibilité de « co-construire, avec les participantes des formes de soutien face aux altérations du sommeil qu'elles vivaient », l'auteure interroge indirectement la nécessité du contre-don dans l'enquête ethnographique (Bouillon, 2006), qui reste à questionner et analyser sur de tels terrains liés à la nuit.

\section{Bibliographie}

Bernard-Hohm M.-C., Raibaud Y. (2012), « Les espaces publics bordelais à l'épreuve du genre », Métropolitiques. En ligne, consulté le 5 décembre 2019. URL : http://www. metropolitiques.eu/Les-espaces-publics-bordelais-a-l.html.

BERTIN S. (2016), Le paysage urbain nocturne: une dialectique du regard entre ombre et lumière, thèse de doctorat en aménagement, Montréal, Université de Montréal.

BEy H. (1991), TAZ (zone d'autonomie temporaire), New York, Autonomédia.

Bianchini F. (1995), « Night Cultures, Night Economies », Planning Practice er Research, vol. 10, n 2, p. 121-126.

Bilhaut A. (2003), « Soñar, recordar y vivir con eso », Estudios atacameños, n² 26, p. 61-70.

Bouillon F. (2000), « Des escales dans la nuit : les snacks égyptiens à Marseille », Les Annales de la recherche urbaine, $\mathrm{n}^{\circ}$ 87, p. 43-51.

Bouillon F. (2006), « Pourquoi accepte-t-on d'être enquêté ? Le contre-don, au cœur de la relation ethnographique ", in F. Bouillon, M. Fresia, V. TAllio, Terrains sensibles. Expériences actuelles de l'anthropologie, Paris, CEA/EHESS, p. 75-95. 
Candela (2017), « Pour une sociologie politique de la nuit », Cultures et Conflits, vol. 1-2, $\mathrm{n}^{\circ}$ 105-106, p. 7-27.

Cauquelin A. (1977), La ville, la nuit, Paris, Presses universitaires de France.

Challeat S. (2010), «Sauver la nuit ». Empreinte lumineuse, urbanisme et gouvernance des territoires, thèse de géographie, Dijon, Université de Bourgogne.

Chatterton P., Hollands R. (2003), Urban Nightscapes: Youth cultures, pleasure spaces and corporate power, New York, Routledge.

Comelli C. (2015), Mutations urbaines et géographie de la nuit à Bordeaux, thèse de doctorat en géographie, Bordeaux, Université de Bordeaux 3.

Deboulet A., Berry-Chikhaoui I., Roulleau-Berger L. (dir.) (2007), Villes internationales. Tensions et réactions des habitants, Paris, La Découverte.

Deleuil J.-M., Toussaint J.-Y. (2000), « De la sécurité à la publicité, l'art d'éclairer la ville », Les Annales de la recherche urbaine, $\mathrm{n}^{\circ}$ 87, p. 52-58.

Didier-FÈvre C. (2018), « La nuit : une nouvelle frontière pour les jeunes des espaces périurbains ? », Géoconfluences. En ligne, consulté le 14 février 2020. URL : http:// geoconfluences.ens-lyon.fr/informations-scientifiques/dossiers-regionaux/franceespaces-ruraux-periurbains/articles-scientifiques/jeunes-periurbains-la-nuit.

Espinasse C., Buhagiar P. (2004), Les passagers de la nuit : vie nocturne des jeunes, Paris, L'Harmattan.

GAISSAD L. (2000), « L'air de la nuit rend libre ? Lieux et rencontres dans quelques villes du sud de la France », Les Annales de la recherche urbaine, n 87, p. 36-42.

Galinier J., Monod A., Bordin G., Fontaine L., Fourmaux F., Ponce J., Salzarulo P., Simonnot P., Therrien M., Zilli I. (2010), dossier « Anthropology of Night: CrossDisciplinary Investigations », Current Anthropology, vol. 51, $\mathrm{n}^{\circ} 6$.

Giordano E. (2017), Paysage lumière : constructions et perceptions. Pour une analyse multi-échelle des géographies lumineuses des villes, thèse de doctorat en géographie, Montpellier, Université de Montpellier 3.

GIORDANo E., GwiAzDZinsKi L. (2018), « La notte urbana, una nuova frontiera per la ricerca geografica in Italia », Rivista Geografica Italiana, vol. 125, n 3, p. 437-452.

Giordano E., Nofre J., Crozat D. (2018), « La touristification de la vie nocturne : une nouvelle frontière pour la recherche sur la nuit urbaine », Cybergeo. En ligne, consulté le 11 janvier 2020. URL : http://journals.openedition.org/cybergeo/29473.

GuÉRIN F. (2016), Enjeux socio-urbains du noctambulisme. Les cas de Paris et Madrid au début $d u$ XXIe siècle, thèse de doctorat en sociologie, Paris, Université Paris-Est.

Gwiazdzinski L. (2005), La nuit dernière frontière de la ville, La Tour d'Aigues, Éditions de l'Aube.

GwiazDzinski L. (2007), Nuits d'Europe. Pour des villes accessibles et hospitalières, Belfort, UTBM. 
Gwiazdzinski L., Pignot L., Saez J.-P. (2019), dossier « Cultures de la nuit : quels enjeux et quels défis ? », L’Observatoire, vol. 1, nº 53.

Gwiazdzinski L., Maggioli M., Straw W. (2019), dossier « Géographies de la nuit/ Geographies of the night/Geografie della notte », Bollettino Della Società Geografica Italiana, vol. $1, \mathrm{n}^{\circ} 2$.

Hadfield P. (2009), Nightlife and Crime: Social Order and Governance in International Perspective, Oxford, Oxford University Press.

Heath T. (1997), « The Twenty four Hour City Concept - A Review of Initiatives in British Cities », Journal of Urban Design, vol. 2, n² 2, p. 193-204.

Jeanmougin H., Bouillon F. (2016), " D'une gentrification inaboutie à une "nouvelle précarisation” ? Continuités populaires et conflits de coprésence dans le centre de Palerme », Lien social et Politiques, $n^{\circ} 77$, p. 103-125.

Jeanmougin H. (2019), "Vie nocturne animée : moteur ou frein à la gentrification ? Conflit d'usage et enjeux contradictoires dans le quartier de la Magione à Palerme », Bollettino della Società Geografica Italiana, vol. 1, nº 2, p. 231-240.

KosLofsky C. (2011), Evening's Empire: A History of the Night in Early Modern Europe, Cambridge, University Press.

Kumar A. (2015), « Cultures of lights », Geoforum, vol. 65, p. 59-68.

LAHIRE B. (2018), L’interprétation sociologique des rêves, Paris, La Découverte.

LIEBER M. (2011), « Le sentiment d'insécurité au prisme du genre. Repenser la vulnérabilité des femmes dans les espaces publics », Métropolitiques. En ligne, consultéle14 février 2020. URL : http://www.metropolitiques.eu/Le-sentiment-d-insecurite-au.html.

Lovatt A., O’Connor J. (1995), " Cities and the Night-time Economy », Planning Practice er Research, vol. 10, $n^{\circ}$ 2, p. 127-134.

MACARIE I. C. (2017), « Invisible denizens: migrant night shift Workers’ fragile possibilities for solidarity in the post-circadian capitalist era », Working Paper Series, vol. 4, Centre for Policy Studies, Central European University. En ligne, consulté le 14 février 2020. URL : https://cps.ceu.edu/publications/working-paper/invisible-denizens-migrantnight-shift-workers-fragile-possibilities.

MALLET S. (2009), Des plans-lumière nocturnes à la chronotopie. Vers un urbanisme temporel, thèse de doctorat en urbanisme, Paris, Université de Paris-Est.

Maldet S. (2014), " Les rythmes urbains de la néolibéralisation. The urban rythms of neoliberalisation ", Justice Spatiale-Spatial Justice, $\mathrm{n}^{\circ}$ 6. En ligne, consulté le 14 février 2020. URL : https://www.jssj.org/article/les-rythmes-urbains-de-laneoliberalisation/.

Mauss M. (1936), « Les techniques du corps », Journal de Psychologie, vol. 32, n 3-4.

Melbin M. (1978), « Night As Frontier », American Sociological Review, vol. 43, n 1, p. 3-22. 
Monod Becquelin A., Galinier J. (2020), "Alors vint la nuit... Terrains, méthodes, perspectives », Ateliers d'anthropologie, n 48. En ligne, consulté le 12 avril 2020. URL : https://journals.openedition.org/ateliers/13380.

Nofre J. (2008), L'Agenda cultural oculta: una deconstrucció de l'oci nocturn de Barcelona i els seus suburbis, thèse de doctorat en géographie, Barcelone, Université de Barcelone.

Nofre J. et al. (2017), "Exploring Nightlife and Urban Change in Bairro Alto, Lisbon », City erCommunity, vol. 16, $n^{\circ}$ 3, p. 237-352.

NOFRE J. et al. (2018), « Tourism, nightlife and planning: challenges and opportunities for community liveability in Barceloneta », Tourism Geographies, vol. 20, n³ 3, p. 377-396.

Nofre J., Eldridge A. (2018), Exploring Nightlife: Space, Society and Governance, Londres, Rowman \& Littlefield International.

O’Connor J., Maury H. (1997), « Donner de l'espace public à la nuit : Le cas des centres urbains en Grande-Bretagne », Les Annales de la recherche urbaine, $\mathrm{n}^{\circ}$ 77, p. 40-46.

PIERONI R. (2017), Institutionnaliser la nuit : géographie des politiques nocturnes à Genève, thèse de doctorat en géographie, Genève, Université de Genève.

Pinçon M., Pinçon-Charlot M. (2000), « Les nuits de Paris », Les Annales de la recherche urbaine, $\mathrm{n}^{\circ} 87$, p. 15-24.

RADICCHI A. (2017), « Beyond the noise: Open source soundscapes - A mixed methodology to analyse, evaluate and plan "everyday" quiet areas ", Proceedings of Meetings on Acoustics, vol. 30, p. 1-14.

Roberts M., Eldridge A. (2009), Planning the Night-time City, Londres, Routledge.

SHAw R. (2015a), « Alive after five: Constructing the neoliberal night in Newcastle upon Tyne », Urban Studies, $n^{\circ}$ 52, p. 456-470.

SHAw R. (2015b), « Night as Fragmenting Frontier: Understanding the Night that Remains in an era of 24/7 », Geography Compass, vol. 9, n 12, p. 637-647.

SHaw R. (2017), « Pushed to the margins of the city: The urban night as a timespace of protest at Nuit Debout, Paris », Political Geography, n 59, p. 117-125.

Straw W. (2015), " Media and the Urban Night », Articulo - Journal of Urban Research, vol. 11. En ligne, consulté le 14 février 2020. URL : http://journals.openedition.org/ articulo/3098.

VAn Liempt I., VAn Aalst I., Schwanen T. (2015), « Introduction : Geographies of the urban night », Urban Studies, vol. 52, n 6, p. 407-421. 